\title{
Correction to: Cross-Graph Representation Learning for Unsupervised Graph Alignment
}

Weifan Wang, Minnan Luo, Caixia Yan, Meng Wang, Xiang Zhao, and Qinghua Zheng

\section{Correction to:}

Chapter "Cross-Graph Representation Learning for Unsupervised Graph Alignment" in: Y. Nah et al. (Eds.): Database Systems for Advanced Applications, LNCS 12113, https://doi.org/10.1007/978-3-030-59416-9_22

In the original edition of this chapter, an institution affiliation was missing in the authors' section for the following authors: Weifan Wang, Minnan Lu, and Qinghua Zheng. The missing institution has been now added. 